\title{
Control of Non-Linear Systems using Fuzzy PI-PD Controller
}

\author{
J.V.G.Rama Rao, K.Ram Charan, A.Rama Krishna, A.Venkata Narayana
}

\begin{abstract}
This paper gives the relationship of control performance of various types of fuzzy controllers, fuzzy (PI-PD) controller, combination of fuzzy PI and PD controller and fuzzy PI-PD controller. The analytical structures of Fuzzy PI-PD controller is deciphered through triangular memebership functions by using mamdani row interference technique and zadeh fuzzy logic and center of gravity defuzzification method. In simulation the control performance of non linear systems for various controllers by taking triangular, Gaussian $g$ bell, trapezoidal memebership functions.
\end{abstract}

Once-over Terms-Fuzzy control structures, loosening up advancement limits, non-rapidly structures, PID controllers.

\section{INTRODUCTION}

For the reason that major achievable use of the peril of the ideal sets [1] to the direct of dynamic plant by system for Mamdani and Assilian [2], there has been mind blowing usually energy for the issue of "Cushioned administer device Engineering". It has been comprehended that it's miles possible to direct planned multifaceted structures clearly with the guide of human administrators who have no investigating of their fundamental fragments, in the meantime as it's far difficult to get the relative with standard controllers. It's miles this reality which has along these lines prompted the pushing toward movement of secure control in a gathering of jobs [3], most remote reason for these applications were set up at the fragment execution of see bosses' commitment.

Starting late, Fuzzy plan of reasoning controllers (FLC), mainly cushioned relative - essential subordinate controllers have been typically used for present day framework inferable from their heuristic nature related pretty genuinely and plentifulness for both direct and non rapidly structures.

Cushioned help controllers may be allocated into three guideline orders: (1) Fuzzy foundation controllers, in which fragile finding and if - reason checks are used without using positive genuine fashions;(2) Fuzzy strategy of reasoning controllers, whose shape is overseen subject to cushioned method for dissuading careful numerical fashions.(three) cushioned reason and show framework joined controllers.

Revised Version Manuscript Received on August 19, 2019.

Dr.J.V.G.Rama Rao, Professor, Dept of Electrical and Electronics Engineering, Bonam Venkata Chalamayya Engineering College, Odalarevu, A.P., India.

K.Ram Charan, Assoc.Professor, Dept of Electrical and Electronics Engineering, Bonam Venkata Chalamayya Engineering College, Odalarevu, A.P., India.

A.Rama Krishna, Assoc.Professor, Dept of Electrical and Electronics Engineering, Bonam Venkata Chalamayya Engineering College, Odalarevu, A.P., India.

A.Venkata Narayana, Asst.Professor, Dept of Electrical and Electronics Engineering, Bonam Venkata Chalamayya Engineering College, Odalarevu, A.P., India.
In vogue PI-PD controllers all around do never again ideal gem acceptably for non-direct structures, and all things considered tremendous and reduce structures which don't have cautious numerical models. To vanquish the ones issues, a class of non-standard fragile PI-PD controllers has been used by us.

The systems of PI - PD and parallel mix of PI and PD checks the key living plans and incredible states of the standard PID controllers, at any rate has a sensible strategy much like the fragile PI and PD controllers. It has 4 tidy cushioned on the up chance that benchmarks for all the PI and PD controllers. So besides the condition of the cushioned PID avows the basic homes and blessings of the standard PID controllers, yet it has eight central warm in the occasion that runs the show.

The general paper must be an undertaking to appreciate what the woolen PI-PD controller is and the way fittingly it can do in test with the elective fleecy PID controllers. Here it has exhibited that the woolen PI-PD controller is higher than the cushioned PID controllers for nonlinear structures, this is certainly not a twisting of the unstable PID Controllers. The unstable PID controllers were proposed for each energetic and non-quickly structures. The fragile PI-PD controller made has some other structure this is by no means, an essential exchange of the common model.

This paper directed as outputs for after, region II portrays the colossal bits of delicate PI-PD controller. Zone III structures fuzzification figuring and fragile direct principles, sort out IV delineates the defuzzification estimation, part V systems clear records yield searching for after and edge VI depicts finally pc beguilements.

\section{FUZZY PI-PD CONTROLLER}

We've used a fragile PI-PD control unit approach, suggested as the subordinate of-yield, a relative vintage clarification of (PI-PD) as showed up in Fig. 1. This methodology is each now and again enchanting if the reference records joins discontinuities. The validation of the unstable PI-PD control standard is rehearsed in stages: In at an opportune time improve the yield of the cushioned PI[4] controller and inside the second step yield of the sensitive $\mathrm{PD}[5]$ controller. The last manage law concretes these character control correctional facility recommendations everything considered in a legitimate manner, as depicted in more detail inside the going with fragments. 


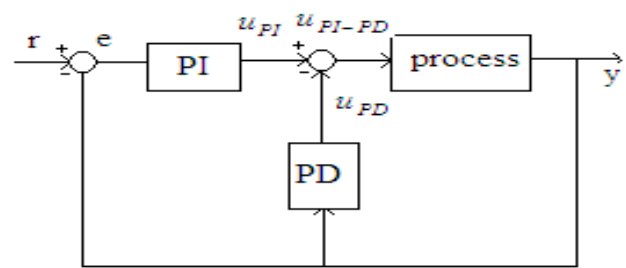

Fig. 1. Conventional PI-PD control system

Combination of the fuzzy PI-PD controller

The complete fuzzy PI-PD control law is obtained with use of Fuzzy PI control law and fuzzy PD control law. The resulting law is

$$
\begin{array}{ll}
u_{P I-P D}(n T)=u_{P I}(n T)-u_{P D(n T)} \\
\text { Or } \quad u_{P I-P D}(n T)=u_{P I}(n T-T)+K_{u P I} \Delta u_{P I}(n T) \\
& +u_{P D}(n T-T)-K_{u P D} \Delta u_{P D}(n T)
\end{array}
$$

"Equation (1)" will be referred to as the fuzzy PI-PD control law throughout the paper.

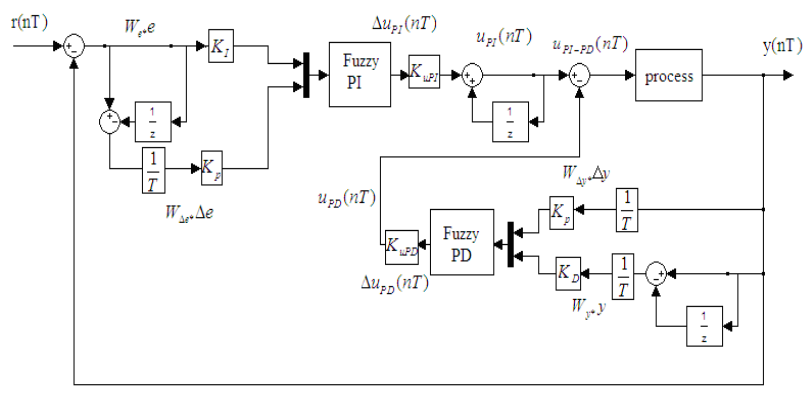

Fig. 2. The fuzzy PI-PD control system

The fragile PI-PD control structure (9) is showed up in Fig. 2. This woolen PI-PD controller is gotten by embeddings the soft PI and the agreeable PD controllers in the standard PI-PD controllers [6].

\section{III.FUZZIFICATION, CONTROL RULE BASE}

on this part, we search for after the run of the mill gadget of tricky controller structure together with fuzzification control standard base system and defuzzification.

\section{A. Fuzzification}

We Fuzzify the PI and PD zones of the fluffy PI-PD control contraption as I should need to think and after that join the favored satisfying control proposals for all of them. the general regulate law yielded (9) is considered at some stage in fuzzification. like the heartless PI controller [7], the enter and the yield club features of the PI piece are endeavored in fig.three. the cushioned PI controller uses inputs, particularly the oversight signal and the charge of trade of messes up sign . the shaggy PI controller has an unmarried yield proposed as the enduring control yield and is showed up as appeared in Fig. 3. The shaggy PD controller has two data sources, especially, ordinary substitute of yield and the charge of exchange of yield . The shaggy PD controller has an unmarried yield known as the unending control yield and is appeared with the guide of structure for . The responsibilities to the thick PD controller must be fuzzified sooner than being continued into the controller. The club features for the 2 inputs and the yield of the controller are exhibited in Fig. 4
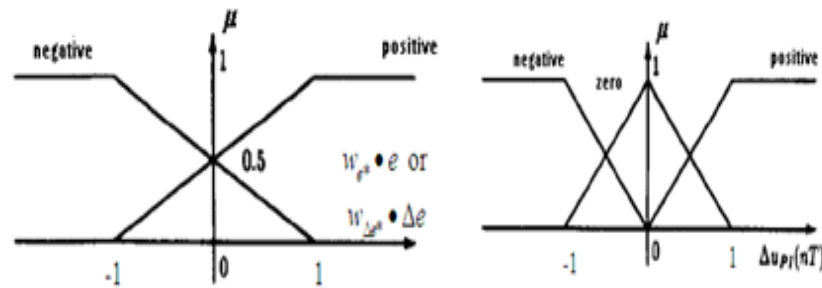

Fig. 3 Membership functions for error, rate of change of error and incremental control output

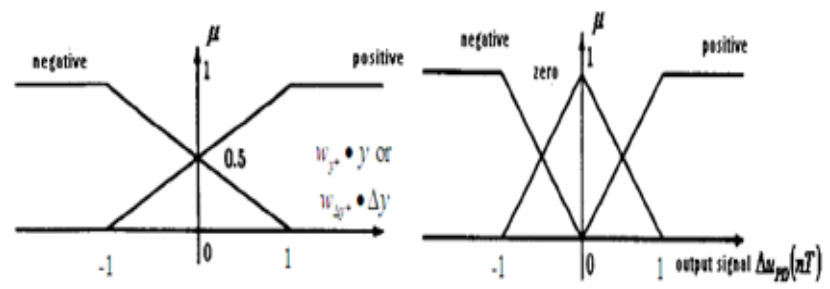

Fig. 4 Membership functions for average output, rate of change of output and incremental control output

\section{B. Control rule base}

Using the starting late referenced theory works, the going with control standards are created for the cushioned PI controller:

R1. If Error is negative AND rate is negative, THEN yield is negative.

$\mathrm{R} 2$. If Error is negative AND rate is certain, THEN yield is zero.

R3. If Error is certain AND rate is negative, THEN yield is zero.

R4. If Error is certain AND rate is certain, THEN yield is certain.

Here "yield" is the warm control action And framework is Zadeh's gifted "And" [1] portrayed by

Basically, from the choice fragments of the woolen PD controller, the going with control benchmarks are used for the PD part.

R5. In case Error is sure AND rate is certain, THEN yield is zero.

R6. In case Error is certain AND rate is negative, THEN yield is certain.

R7. In case Error is negative AND rate is certain, THEN yield is negative.

R8. In case Error is negative AND rate is negative, THEN yield

is zero.

In the above standards, "PD yield" is the woolen PD control yield . Also, various terms are depicted correspondingly to the PI part.

For the parallel mix of agreeable PI and PD controller, the measures for Fuzzy PI part are R1-R4 and the guidelines for Fuzzy PD part are R5-R8.

These eight standards absolutely yield the control rehearses for the cushioned PID control law. 
R1. IF e $e_{p} \in e_{p} \bullet n$ and $e_{I} \in e_{I} \bullet n$ and $e_{D} \in e_{D} \bullet n$

R2. IF e $e_{p} \in e_{p}^{\bullet} n$ and $e_{I} \in e_{I} \bullet n$ and $e_{D} \in e_{D} \bullet p$ THEN $\Delta u \in \Delta u \bullet n$

R3. IF $\mathrm{e}_{p} \in e_{p} \bullet p$ and $e_{I} \in e_{I} \bullet n$ and $e_{D} \in e_{D} \bullet n$ THEN $\Delta u \in \Delta u \bullet n$

R4. IF $\mathrm{e}_{p} \in e_{p} \bullet p$ and $e_{I} \in e_{I} \bullet n$ and $e_{D} \in e_{D} \bullet p$ THEN $\Delta u \in \Delta u \bullet p$

R5. IF $\mathrm{e}_{p} \in e_{p} \bullet n$ and $e_{I} \in e_{I} \bullet p$ and $e_{D} \in e_{D} \bullet n$ THEN $\Delta u \in \Delta u \bullet n$

R6. IF e $e_{p} \in e_{p} \bullet n$ and $e_{I} \in e_{I} \bullet p$ and $e_{D} \in e_{D} \bullet p$ THEN $\Delta u \in \Delta u \bullet p$

R7. IF e $\in e_{p} \bullet p$ and $e_{I} \in e_{I} \bullet p$ and $e_{D} \in e_{D} \bullet n$ THEN $\Delta u \in \Delta u \bullet p$

R8. IF e $e_{p} \in e_{p} \bullet p$ and $e_{I} \in e_{I} \bullet p$ and $e_{D} \in e_{D} \bullet p$ THEN $\Delta u \in \Delta u \bullet p l$

The " picture addresses the "titanic" regard in the looking

\section{IV.DEFUZZIFICATION}

In the defuzzification experience, for each the fragile PI and PD controllers, the expectedly used "focal factor of gravity" gadget [4], [6] is used to defuzzify the contemporary control of the cushioned control rule. The "factor of blending of mass" included substances for the defuzzification examines as:

\section{ANALYTICAL ENTER-OUTPUT RELATIONSHIP}

In light of using Zadeh AND Operator, the data region should be circulated phenomenal zones so that in the midst of each recognize an astounding hunting down can be gotten for each agreeable standard a bit of the 2 confirmation cutoff focuses being ANDed. First recall a close old forerunner segments which incorporate reinforce works the most remote point of view on which the confirmation regard is unclear among interest explanations behind restriction is gotten by using the usage of empowering them to move to. On the point in the meantime as the cutoff is open it is insignificant to make comprehension of which control has a claim to fame with which IC sign up the estimation of and using one a few botch and charge in both IC

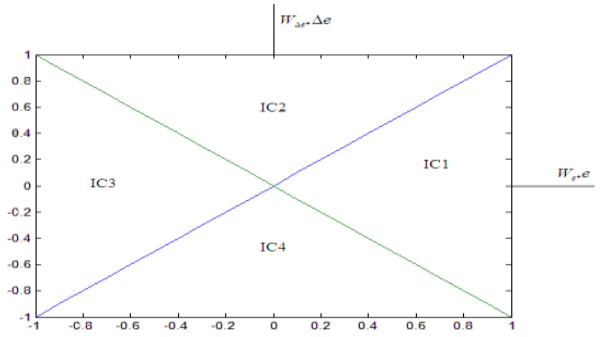

Fig. 6. Regions of the fuzzy PI controller input-combination values THEN $\Delta u \in \Delta u \bullet n l$ of either positive (p) or negative (n).

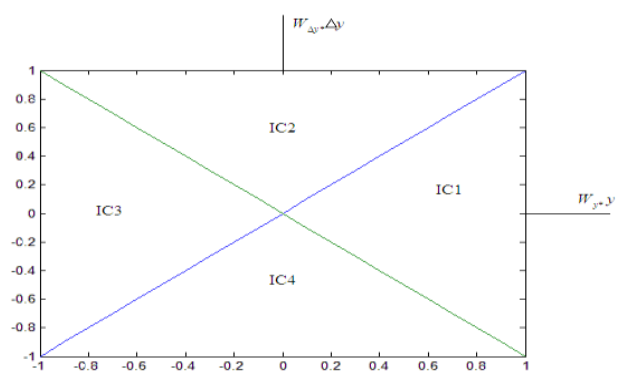

Fig. 7. Regions of the fuzzy PD controller input-combination values

Superimposing the four individual data zone divisions to plot standard enter a locale branch for all proposals in cushioned PI controller is showed up in fig [6] and for soft PD controller are endeavored in fig [7]. The control rules for the shaggy PI controller (R1)- (R4), with choice joins and enter-rigid (IC) areas together, are used to consider sensible cushioned control laws for each locale. walking around procedures everything being similar, we achieve the going with plan for the 4 IC areas are appeared in work a zone

I.TABLE I Input Spaces of Fuzzy PI Controller

\begin{tabular}{|c|c|}
\hline IC1 & $\frac{W_{\Delta u}\left[W_{e^{*}} e+W_{\Delta e^{*}} \Delta e\right]}{4-2 W_{e^{*}} e}$ \\
\hline IC2 & $\frac{W_{\Delta u}\left[W_{e^{*}} e+W_{\Delta e^{*}} \Delta e\right]}{4-2 W_{\Delta e^{*}} \Delta e}$ \\
\hline IC3 & $\frac{W_{\Delta u}\left[W_{e^{*}} e+W_{\Delta e^{*}} \Delta e\right]}{4+2 W_{e^{*}} e}$ \\
\hline IC4 & $\frac{W_{\Delta u}\left[W_{e^{*}} e+W_{\Delta e^{*}} \Delta e\right]}{4+2 W_{\Delta e^{*}} \Delta e}$ \\
\hline
\end{tabular}

So besides the defuzzification of the soft PD controller searches for after an equivalent technique as depicted above for the PI territory, with the amazing case that the experiences standard for this case are exceptional. We found the endeavor furthest reaches of the typical risk of the records signal (given Fig. Four) over the even - focus and the hypothesis most distant extents of the unfaltering control posted notice over the vertical - flip as showed up in Fig. 7. Like the conditions in above, we use the characteristics and exercise the quickly line plans have been given from the geometry of Fig. 7. Is seemed, by all accounts, to be TABLE II.

TABLE II Input spaces for Fuzzy PD Controller

\begin{tabular}{|c|c|}
\hline IC1 & $\frac{W_{u}\left[W_{e^{*}} e+W_{\Delta e^{*}} \Delta e\right]}{4-2 W_{e^{*}} e}$ \\
\hline IC2 & $\frac{W_{u}\left[W_{e^{*}} e+W_{\Delta e^{*}} \Delta e\right]}{4-2 W_{\Delta e^{*}} \Delta e}$ \\
\hline IC3 & $\frac{W_{u}\left[W_{e^{*}} e+W_{\Delta e^{*}} \Delta e\right]}{4+2 W_{e^{*}} e}$ \\
\hline IC4 & $\frac{W_{u}\left[W_{e^{*}} e+W_{\Delta e^{*}} \Delta e\right]}{4+2 W_{\Delta e^{*}} \Delta e}$ \\
\hline
\end{tabular}


Therefore from TABLE I \& TABLE II, we have derived the possible 16 analytical expressions for Fuzzy PI-PD controller and are listed in TABLE III.

TABLE III Analytical expressions for Fuzzy PI-PDController

\begin{tabular}{|c|c|}
\hline S. No & Analyoical experessions for Fuzzy PI-PD consoller \\
\hline 1 & $\frac{W_{\Delta u}\left[W_{\alpha} e+W_{\mu}-\Delta e\right]}{4-2 W_{\alpha} e}-\frac{W_{u}\left[W_{\rho} e+W_{\Delta s} \Delta e\right]}{4-2 W_{e} e}$ \\
\hline 2 & 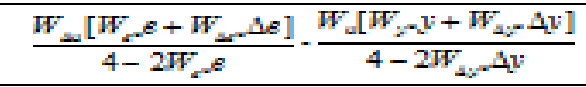 \\
\hline 3 & $\frac{W_{d}\left[W_{j}-y+W_{\Delta y}-\Delta y\right]}{4+2 W_{j} y}-\frac{W_{d}\left[W_{;} y+W_{\Delta y}-\Delta y\right]}{4+2 W_{j} y}$ \\
\hline 4 & 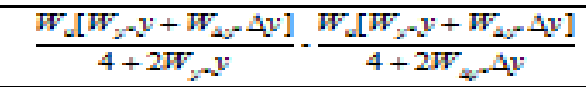 \\
\hline 3 & $\frac{W_{\Delta u}\left[W_{\alpha} e+W_{\Delta s} \Delta e\right]}{4-2 W_{\Delta \alpha} \Delta e}-\frac{W_{\mathrm{u}}\left[W_{e} e+W_{\Delta \alpha} \Delta e\right]}{4-2 W_{\alpha} e}$ \\
\hline 6 & $\frac{W_{\Delta u}\left[W_{\alpha} e+W_{\Delta-\Delta e}\right]}{4-2 W_{\Delta} \Delta e}-\frac{W_{d}\left[W_{j}-y+W_{\Delta j}-\Delta y\right]}{4-2 W_{\Delta,} \Delta y}$ \\
\hline T & 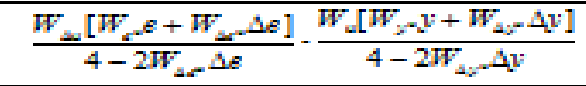 \\
\hline 3 & 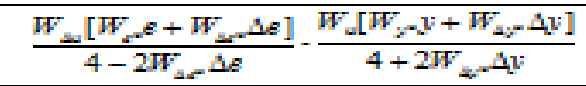 \\
\hline 9 & $\frac{W_{\mu u}\left[W_{\alpha} e+W_{\Delta}-\Delta e\right]}{4+2 W_{\alpha} e}-\frac{W_{\mathrm{u}}\left[W_{\alpha} e+W_{\Delta \alpha}-\Delta e\right]}{4-2 W_{\alpha} e}$ \\
\hline 10 & 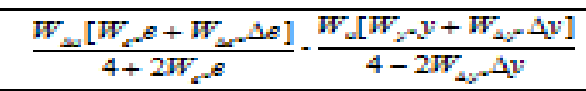 \\
\hline 11 & 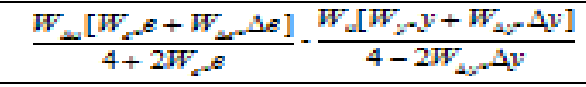 \\
\hline 12 & 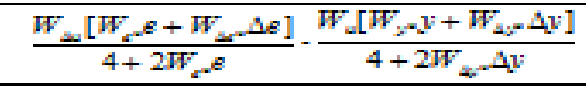 \\
\hline 13 & $\frac{W_{\Delta u}\left[W_{\alpha} e+W_{\Delta s} \Delta e\right]}{4+2 W_{\Delta \alpha} \Delta e}-\frac{W_{u}\left[W_{e} e+W_{\Delta s} \Delta e\right]}{4-2 W_{\alpha} e}$ \\
\hline 14 & 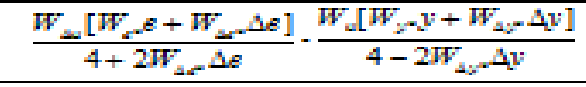 \\
\hline 15 & 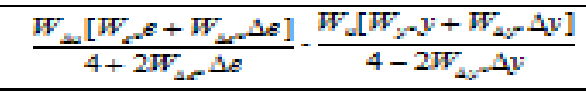 \\
\hline 16 & $\frac{W_{\Delta u}\left[W_{\alpha} e+W_{\Delta-\Delta e}\right]}{4+2 W_{\Delta \alpha} \Delta e}-\frac{W_{d}\left[W_{z}-y+W_{\Delta y}-\Delta y\right]}{4+2 W_{\alpha}-\Delta y}$ \\
\hline
\end{tabular}

\section{COMPUTER SIMULATIONS\& RESULTS}

As a first point of view consider a non-direct structure depicted by condition $=0.0001+\mathrm{u}(\mathrm{t})$ and the unit step Responses are showed up in Fig. 8 to Fig. 12.pc duplications are created for the Fuzzy PI-PD controller,Parallel mix of Fuzzy PI+PD Controller , and three enter Fuzzy PID Controller then as triangular, Gbell, trapezoidal, pi, and Gaussian club aptitudes in every datum and yield are used .

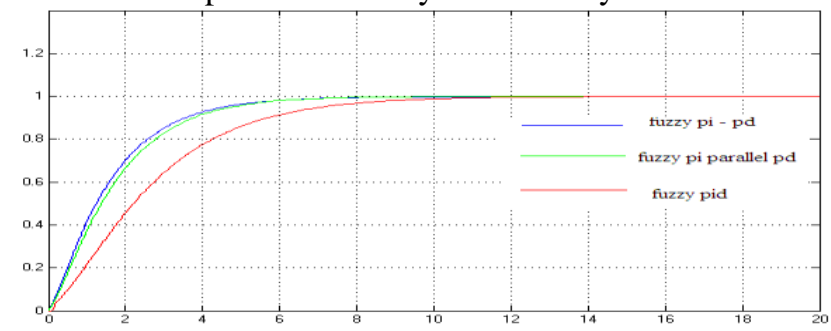

Fig. 8 "Triangular" membership function

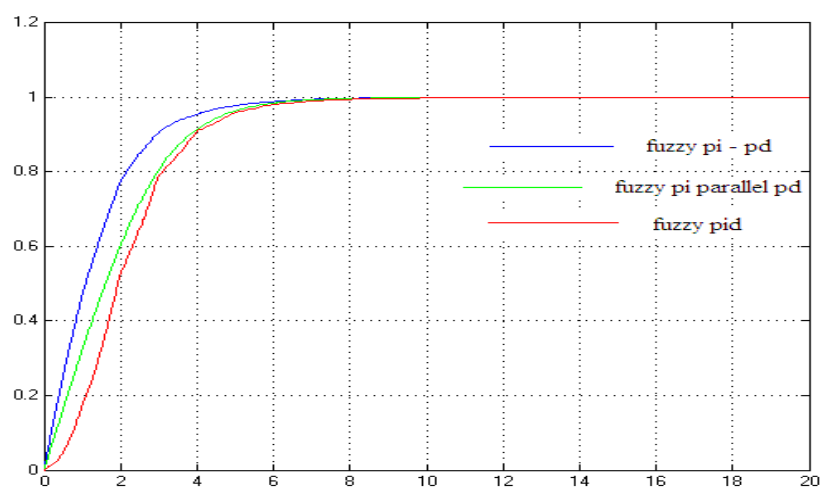

Fig. 9 "Gbell” membership function

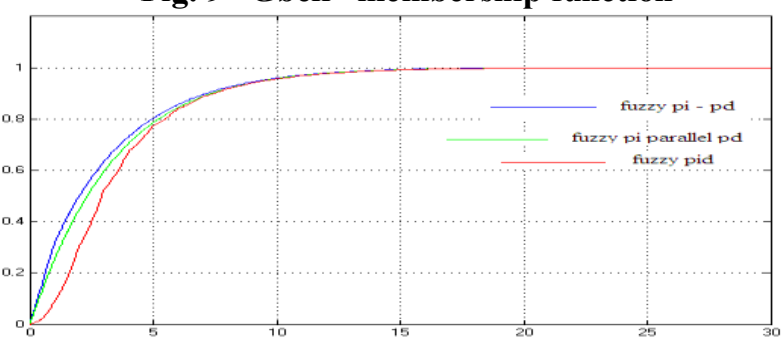

Fig. 10 For "Trapezoidal" membership function

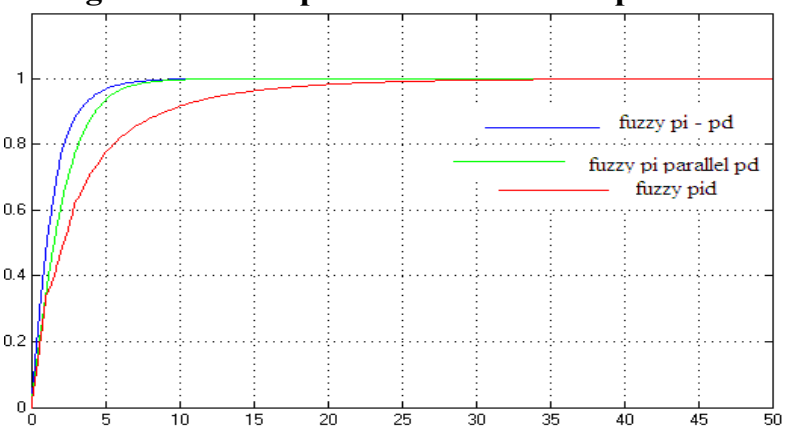

Fig. 11 "Pi" membership function

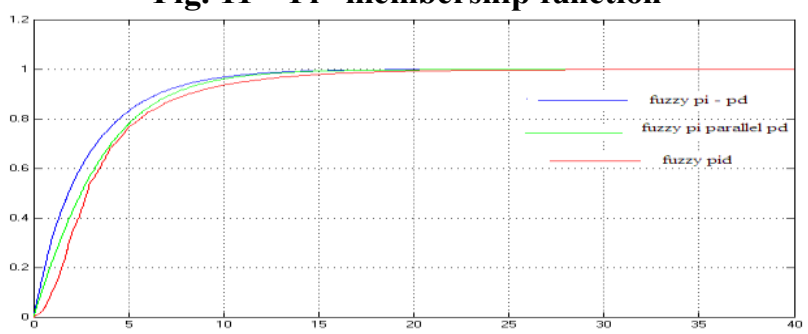

Fig. 12 "Gaussian" membership function

As a second example consider a non-linear system described by the equation $=-\mathrm{y}(\mathrm{t})++\mathrm{u}(\mathrm{t})$ and unit step responses are shown in Fig. 13 to Fig. 17,with different membership functions

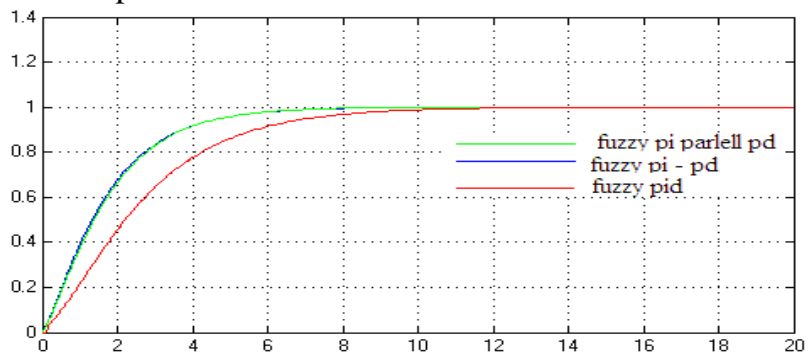

Fig. 13 "Triangular" membership function 


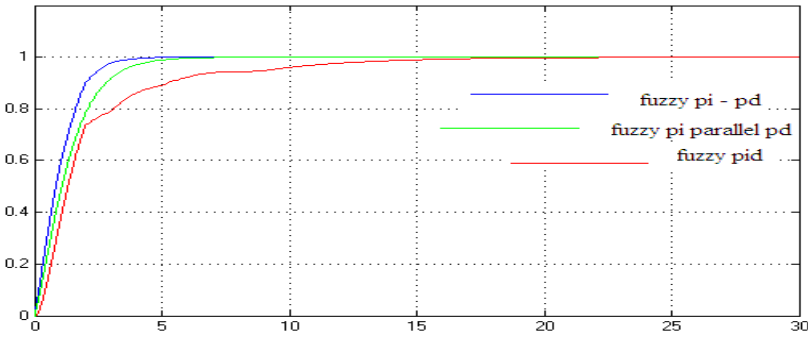

Fig. 14 "Gbell" membership function

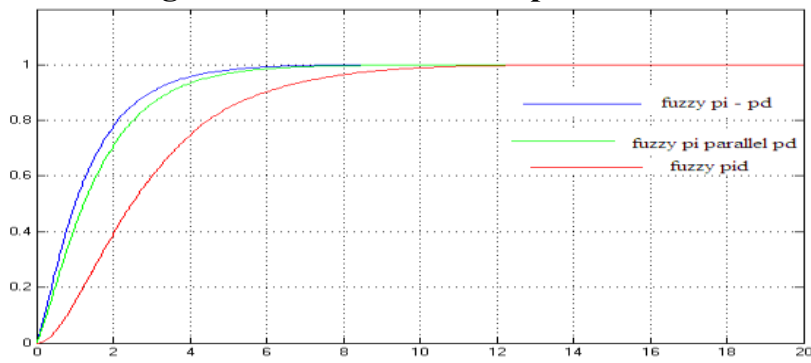

Fig. 15 "Trapezoidal membership function

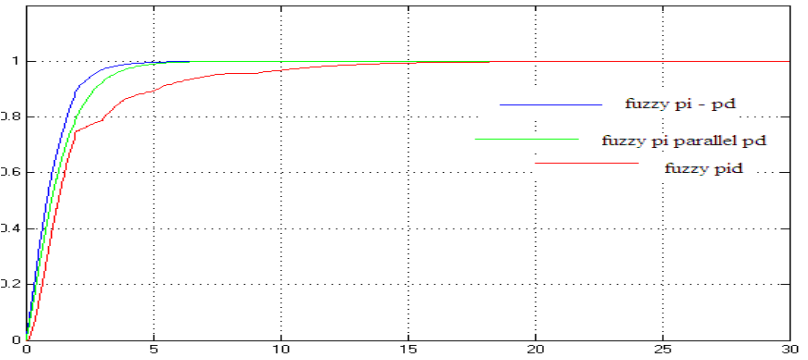

Fig. 16 For "Pi" membership function

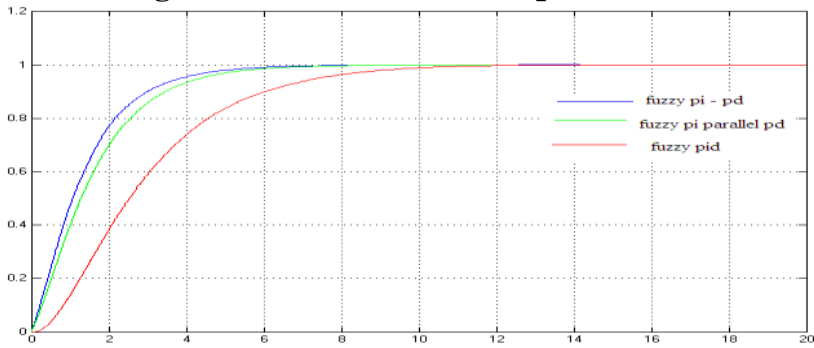

Fig. 17 For "Gaussian" membership function

Next consider the non-linear system described by the equation

$$
\dot{y}=\mathrm{y}(\mathrm{t})+\mathrm{u}(\mathrm{t})+\sin ^{2}(\sqrt{|y(t)|}) \text {. }
$$

The unit step responses for this non-linear system are shown in Fig. 18 to Fig. 22, with different membership functions.

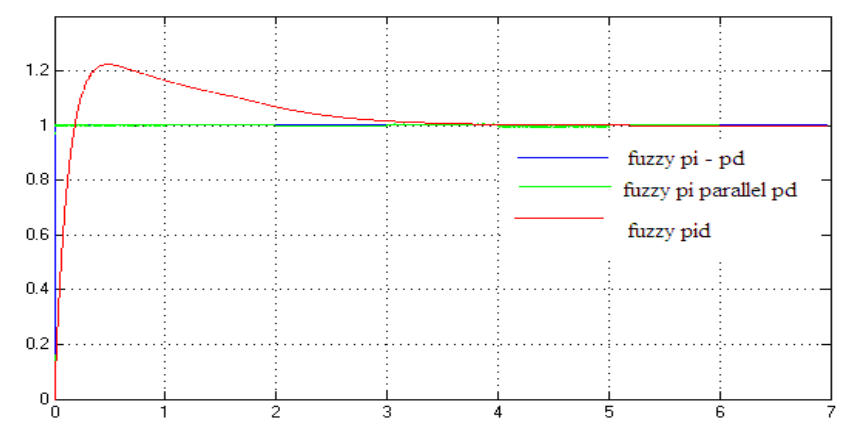

Fig. 18 "Triangular" membership function

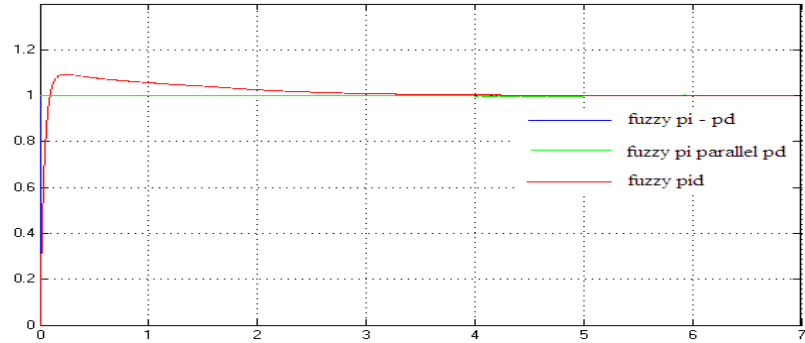

Fig. 19 "Gbell” membership function

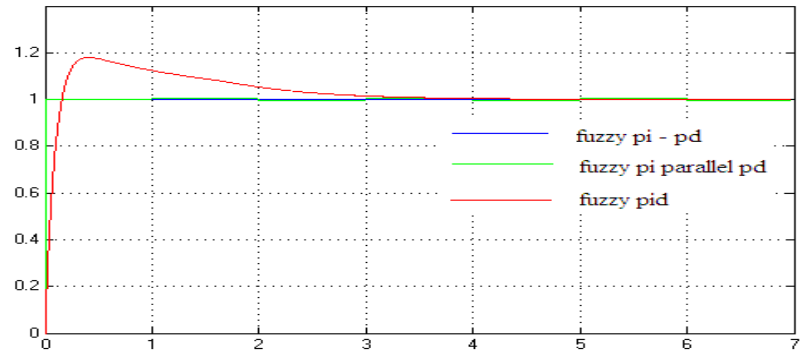

Fig. 20 "Trapezoidal" membership function

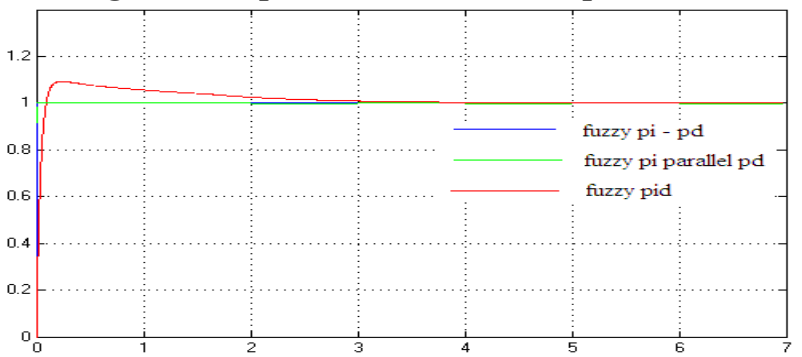

Fig.21"Pi" membership function

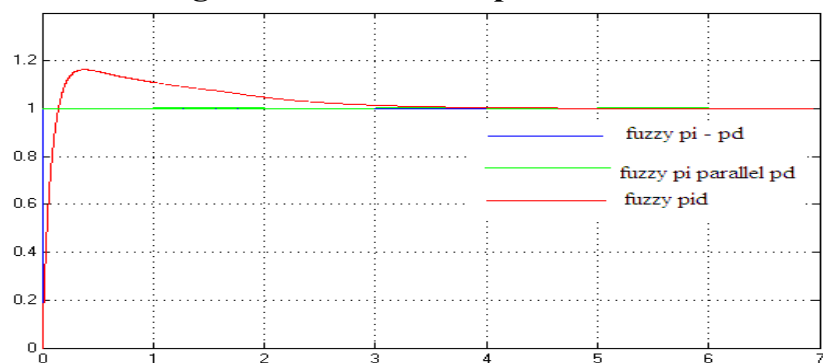

Fig. 22 “Gaussian" membership function

\section{VII.CONCLUSIONS}

We have given sketched out the association standard of a fuzzy PI-PD controller and beside the reasonable records and yield people from the family are initiated the use of triangular affirmation limits. The smooth PI-PD controller is a discrete time of the standard PI-PD controller. The results show that the smooth PI-PD controller reveals the standard direct execution over parallel combination of cushioned PI+PD controller. The outcomes display that the parallel combination of agreeable PI+PD controller is higher than 3 data Fuzzy PID Controller.

\section{REFERENCES}

1. Zadeh, L. A (1965). Tricky units. Inf. Control, 8 , pp-338-353.

2. E.H Mamdani, S. Assilian. A test in semantic association with a sensitive procedure of reasoning controller, 
3. internat. J. Particular contraption stud.7 (1)(1974). Pp1-13.

4. Sugeno, M, (1985). Mechanical employments of sensitive administer, Amsterdam: North-Holland.

5. G. Chen and H. Yang, "on the steadiness of cushioned PI manage structures," in proc. 1/3 internat. Conf. On Fuzzy sound judgment applications, Dec 1993,pp.128-133.

6. H.A. Malki, H.Li, and G. Chen, "New affiliation and achievement examination of smooth concerning subordinate administer systems," IEEE Transactions on Fuzzy structures, vol. 2, no.4, pp 245-253, 1994.

7. D.Misir, H.A.Malki and G.Chen, "Plan and fundamental of a Fuzzy surveying key subordinate controller," Fuzzy set and structures, Vol.Seventy nine, pp 297-314, 1996.

8. H. Ying, W. Siller and J.J.Buckley, "great control thought: A non rapidly case", Automatica, Vol.26, PP.513-520, 1990. 